\title{
Morphogenesis: a focus on marine invertebrates
}

\author{
Zhiyi Lv ${ }^{1,2,3,4} \cdot$ Qiongxuan Lu' ${ }^{1}$ Bo Dong ${ }^{1,2,3}$
}

Received: 3 July 2019 / Accepted: 23 October 2019 / Published online: 25 November 2019

(C) The Author(s) 2019

\begin{abstract}
Morphogenesis is a process describing how the shapes of living tissues and bodies are created during development. Living and fossil organisms exhibit enormously diverse tissue architecture and body forms, although the functions of organs are evolutionally conserved. Current knowledge reveals that relatively conserved mechanisms are applied to control development among different species. However, the regulations of morphogenesis are quite diverse in detail. Animals in the ocean display a wide range of diversity of morphology suitable for their seawater environment. Nevertheless, compared with the intensive studies on terrestrial animals, research on marine animal morphogenesis is still insufficient. The increasing genomic data and the recently available gene editing methods, together with the fast development of imaging techniques, quantitative analyses and biophysical models, provide us the opportunities to have a deeper understanding of the principles that drive the diverse morphogenetic processes in marine animals. In this review, we summarize the recent studies of morphogenesis and evolution at molecular, cellular and tissue levels, with a focus on three model marine animals, namely ascidians, sea urchins and sea anemones.
\end{abstract}

Keywords Ascidian $\cdot$ Morphogenesis $\cdot$ Sea anemone $\cdot$ Sea urchin

\section{Introduction}

Morphogenesis is one of the most exciting phenomena in living organisms, and the mechanisms underlie the formation of diverse morphologies have fascinated biologists for over a century. At the cellular level, morphogenesis is achieved by cell division and death, cell shape change, cell migration and cell rearrangement. The cells get the instructions for what should be done from a signaling cascade starting from morphogens. Morphogens are diffusible molecules that specify

Edited by Jiamei Li.

Bo Dong

bodong@ouc.edu.cn

1 Ministry of Education Key Laboratory of Marine Genetics and Breeding, College of Marine Life Sciences, Ocean University of China, Qingdao 266003, China

2 Laboratory for Marine Biology and Biotechnology, Qingdao National Laboratory for Marine Science and Technology, Qingdao 266237, China

3 Institute of Evolution and Marine Biodiversity, Ocean University of China, Qingdao 266003, China

4 Institute for Developmental Biochemistry, Medical School, University of Göttingen, 37077 Göttingen, Germany cell fates in a concentration-dependent manner, thereby setting up the patterning of a given tissue (Lawrence 2001). The term "morphogen" was coined by Alan Turing in his seminal paper (Turing 1953). Decades later, genes encoding morphogens were identified by Christiane Nüsslein-Volhard and Eric Wieschaus in the famous "Heidelberg screen" (Nüsslein-Volhard and Wieschaus 1980), which was a largescale genetic screen for the genes essential for Drosophila embryogenesis. Further genetic cloning and molecular characterizations revealed that most morphogens are secreted signaling proteins, such as Wnt, fibroblast growth factor (FGF) and Hedgehog (Lawrence 2001). The morphogens bind to specific receptors, thereby activating or deactivating certain genetic circuits in defined domains of the developing tissue. Those genetic circuits do not control cell shape directly. Rather, cell and tissue shape change is executed by cellular mechanical machinery (including actomyosin networks, adherens junctions, cell polarity complexes) in the cell cortex ("Box 1"). The genetic circuits activate or suppress the expression of proteins, such as guanine nucleotide exchange factors (RhoGEF) (Kölsch et al. 2007) and phosphatase and tensin (PTEN) (Bardet et al. 2013), which then modulate the activities or properties of the cellular machinery spatiotemporally, to determine exactly when, where and how a specific tissue shape forming will take place (Fig. 1a). 
Upon the arrival of instructions, the cells undergo remodeling of their cytoskeletons and junctions, leading to the coordination among the cells at tissue level by chemical-mechanical signals. As a result, the tissue achieves the designated shape. This indicates that morphogenesis can be analyzed at different levels: the molecular level, the cellular level and the tissue level (Fig. 1b). The collective behavior of the lower level in the hierarchy leads to the emergence of new properties. These emergent properties and their functions, however, are not seen at the lower level.

Mechanical forces generated by the cellular machinery propagate through transmembrane receptors to neighboring cells and the extracellular matrix. The integration of these

A

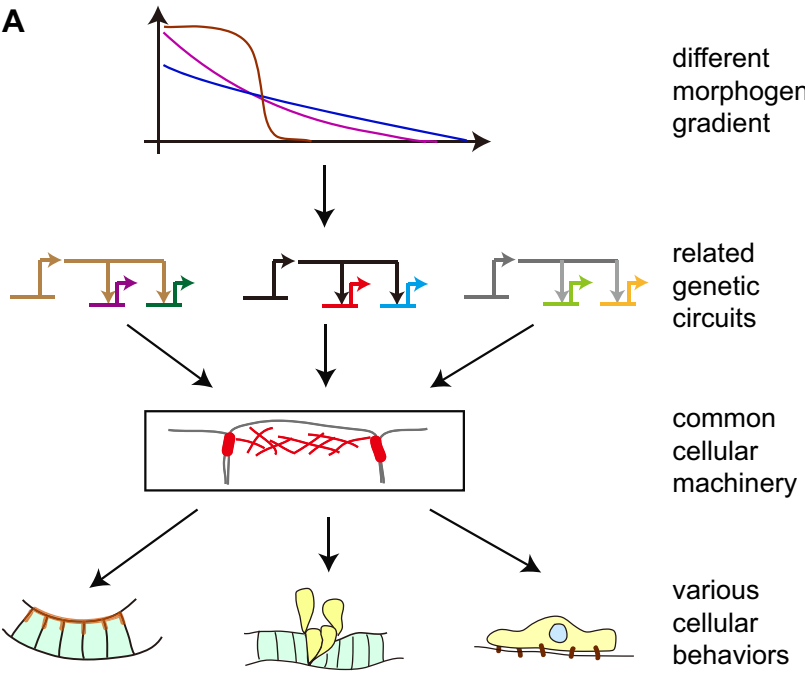

B

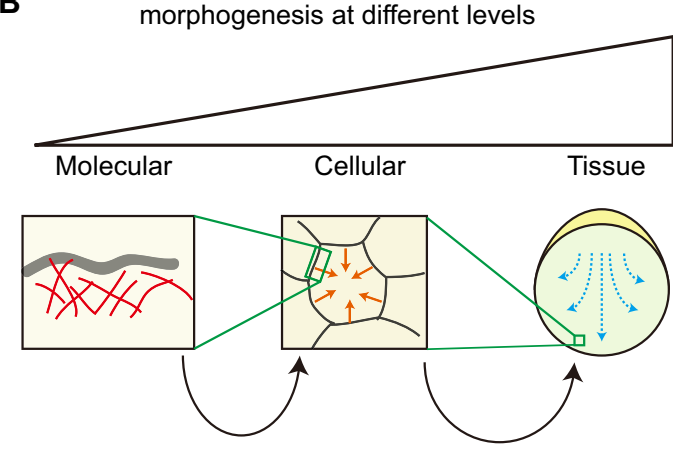

Collective behaviors from lower level lead to the emergence of the new features at higher level.

Fig. 1 Overview of morphogenesis. a Morphogen concentration determines the morphogenesis in certain regions of embryos via activating or inactivating related genetic circuits, which control the cellular mechanical machinery at the cell cortex. Cell cortex functions as a hub, promoting cellular apical constriction, epithelia-mesenchymal transition, or migration to sculpture the tissue, depending on the developmental contexts. b Morphogenesis occurs at different levels. The interactions of the elements at lower level lead to the emergence of new features at higher level forces on tissue level drives morphogenetic events, such as tissue invagination, stratification and elongation. There is growing evidence that the information flow from morphogen to tissue architecture is not unidirectional. Rather, a feedback loop exists between morphogens and the tissue shaping process. After forming a certain shape, the tissue in turn affects both the distribution of morphogens and cell fate (Averbukh et al. 2014; Shyer et al. 2015). However, the feedback mechanisms remain elusive.

Oceans provide $71 \%$ of the living space on our planet, and an estimated 243,000 accepted marine species in higher taxa are found in oceans (Costello and Chaudhary 2017), most of which are invertebrates. Marine invertebrates exhibit a wide range of developmental procedures and diverse forms of body plan, from the simple, soft sac-like animal Cnidaria to Urochordates (tunicates), the closest living invertebrate relative of the vertebrates. Marine invertebrates provide a great source of interesting morphogenic cases, for example, the remarkable morphological mollusk shells (McDougall and Degnan 2018) and the intricate polychaete germline morphogenesis (Brubacher and Huebner 2009). Different developmental modes are found in marine invertebrates: some animals undergo direct development without feeding larval stage in the life cycle, and other species contain a feeding larval stage and instead undergo indirect development. The morphologies and the wide range of tissue remodeling (Johnson et al. 2019) during the metamorphosis are also of great interest. To develop a comprehensive understanding of the mechanism underlying morphogenesis in marine invertebrates, it is important to focus on major marine model organisms, which are genetically and visually trackable in vivo. Recent advances in live imaging techniques, genetical perturbation, and quantitative analysis have facilitated a better understanding of marine invertebrate morphogenesis at molecular, cellular and tissue levels. In this review, we discuss these progresses, focusing on the recent discoveries and open questions in the areas of cellular dynamics during ascidian, sea urchin and sea anemone morphogenesis (see “Box 2").

\section{Box 1. The molecular machinery of morphogenesis}

Beneath the plasma membrane is a thin network made of actin filaments, cross-linkers and motor protein nonmuscle myosin II. The motor activity generates the contractile force in the cortical network under the regulation of several signals. The cortex is attached to the cell membrane by ERM proteins (Fehon et al. 2010); therefore, the structures and the properties of the cortex determine the cell shape. Cell polarity refers to spatial difference in cellular components in one cell. Cell polarity plays an important role 
during morphogenesis. Epithelial cells display apical-basal polarity. The establishment and maintenance of cell polarity involve positive and negative feedback between multiple polarity molecules, in which the Par-3/Par-6/aPKC complex plays a fundamental role. Adherens junctions (AJs) mediate cell-cell junction via homophilic trans-interaction of cadherins molecules from neighboring cells. Inside the cell, AJs bind to actin filaments, therefore the actomyosin contraction can be transmitted via AJs across multiple cells.

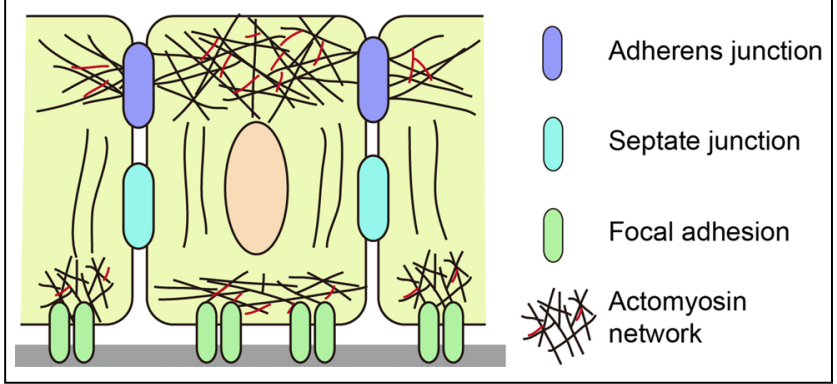

\section{Box 2. Sea urchins, sea anemones and ascidians}

Compared with their terrestrial and freshwater counterparts, the molecular and cellular biology of marine animals is poorly known, despite their large number of species and high diversity of morphology. Owing to the development of genome sequencing and the live imaging techniques, the marine animals, especially sea urchins (McClay 2011), sea anemones (Technau and Steele 2011) and ascidians (Lemaire 2011), have emerged as powerful model organisms for research on evolutionary developmental genetics and morphogenesis. Sea anemones belong to the phylum Cnidaria, which have only two germ layers, the outer ectoderm and the inner endoderm or gastrodermis which lines the gut cavity. This prominent feature makes the starlet sea anemone, Nematostella vectensis, an ideal model for studies on the evolution of the mesoderm (Steinmetz et al. 2017) and bilaterians (He et al. 2018). Sea urchins belong to the phylum Echinodermata. Their embryos have been used as a model system to address many questions in developmental biology since the 1800s. Sea urchins can be maintained easily in laboratories. Their optical transparency makes sea urchins an attractive model for in vivo imaging. The launch of its genome sequence has made the sea urchin a popular model organism for the study of developmental biology (Sea Urchin Genome Sequencing Consortium et al. 2006). Ascidians, known as sea squirts, are invertebrate chordates, which belong to the subphylum Urochordata/Tunicata. They have a notochord at the larval stage, but they lack vertebrae in their life cycle. Ascidian larvae are free-swimming tadpoles, whereas, after metamorphosis, the tail undergoes regression associated with notochord degeneration, and the animal settles on a hard surface. The genome had been sequenced by 2002 (Dehal et al. 2002). Research on ascidians has shed light on notochord evolution (Reeves et al. 2017) and the origin of important vertebrate tissues/organs, such as the neural crest (Abitua et al. 2015; Stolfi et al. 2015).

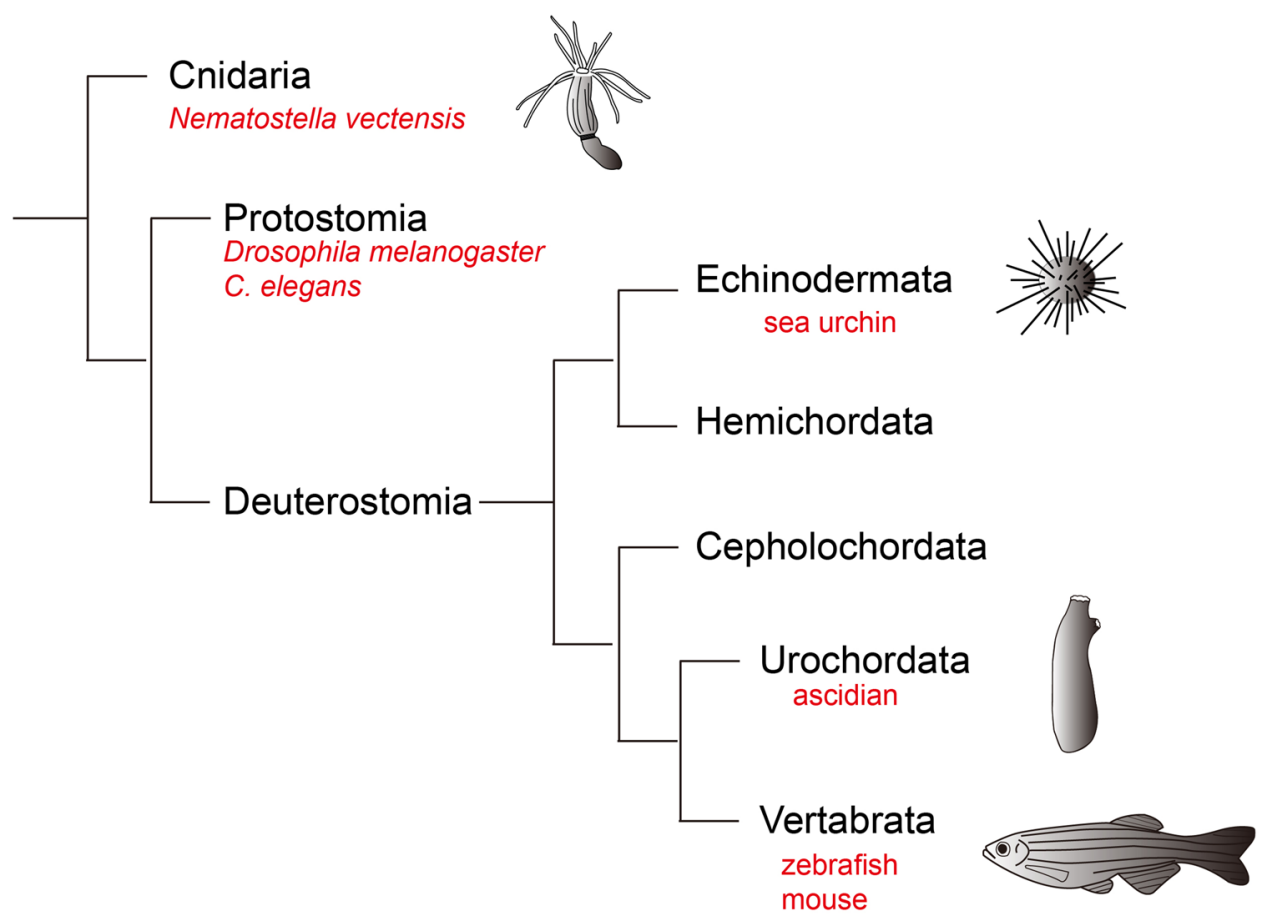


Fig. 2 Actin-based force generation apparatus. a Actin polymerization leads to protrusive activity, i.e., lamellipodia and filopodia. b Actomyosin networks have the ability to contract via different mechanisms (see main text). $\mathbf{c}$ The F-actin attaches to cell-cell or cell-ECM contacts
A

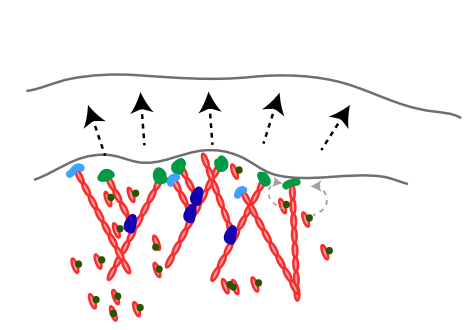

\section{- Arp2/3 capping protein G-actin addtion}

B

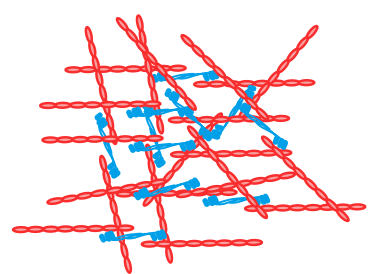

$\infty$ myosin
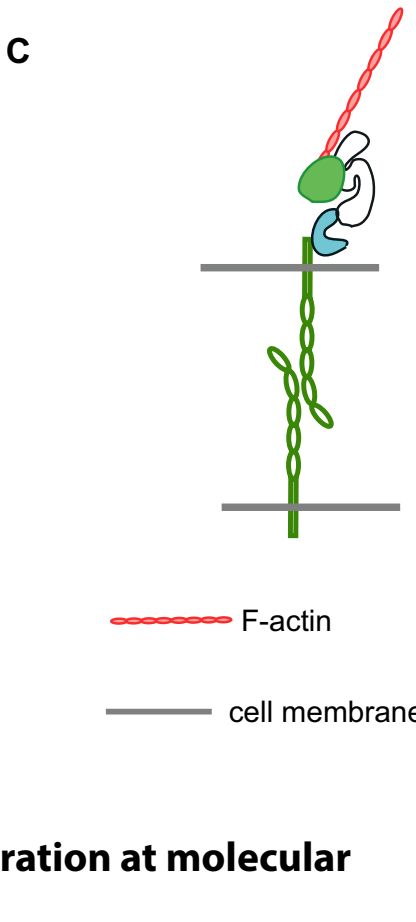
level

Morphogenesis is a well-known self-organized process, driven by forces that are generated and integrated within tissues (Gilmour et al. 2017; Heisenberg and Bellaïche 2013). These forces can be generated by motor activity on polymers, elongation of actin and microtubule filaments, osmotic pressure and other mechanisms (Mammoto and Ingber 2010). Two different strategies are utilized for actin-based force generation: (1) actin filament assembly beneath the plasma membrane and (2) actomyosin network contraction (Fig. 2a, b). Although actin monomers can spontaneously polymerize into filaments in vitro (Pollard and Cooper 2009), living cells control actin filament assembly tightly. Profilin binding to actin monomers hampers actin self-assembly (Theriot and Mitchison 1993), and the capping proteins bind to barbed ends of existing actin filaments to restrict filament growth (Borisy and Svitkina 2000). Only in the presence of actin nucleator and elongation factors such as Arp2/3, formin and 
Ena/VASP protein can actin filaments grow. At the cellular level, actin filament-based forces are wildly used for cell shape change, migration and division. The growth of actin filaments pushes the plasma membrane to form protrusive structures at the leading edges of cells, such as lamellipodia and filopodia (Rottner and Schaks 2019). Lamellipodia are thin-layer protrusive structures at the front of spreading and migrating cells, which contain two-dimensional actin networks. Lamellipodia are found in motile cells in various contexts, including wound healing in sea anemones (Kamran et al. 2017), archenteron elongation in sea urchins (Kominami and Takata 2004) and notochord morphogenesis in ascidians (Munro and Odell 2002). Filopodia are thin, tubular extensions of the plasma membrane with parallel actin bundles inside. Filopodia structures were observed in the second mesenchymal cells of sea urchins (Gustafson and Wolpert 1961; Miller et al. 1995), in the tunic of the ascidian Botryllus schlosseri (Izzard 1974) and in the endoderm of sea anemones (Kraus and Technau 2006). Cytoneme is a type of filopodium that can deliver signaling proteins to target cells or receive signaling proteins from other cells (Kornberg and Roy 2014). It is highly likely that filopodia play a role as cytonemes in the developmental processes mentioned above. However, further molecular characterization is required.

The second strategy is the usage of actomyosin networks. Nonmuscle myosin II (referred to hereafter as myosin) molecules assemble into bipolar minifilaments with two motor domains at the ends, embedded in actin networks that can be organized monoscopically in 2D networks (Chugh and Paluch 2018), rings (Abreu-Blanco et al. 2012) or linear bundles (Monier et al. 2010). The motor activity allows myosin to move on F-actin, thereby generating contractile forces. Actomyosin contractility plays an indispensable role in numerous biological processes, such as apical constriction, body axis formation in Caenorhabditis elegans (Munro et al. 2004) and cell junction remodeling (Rauzi et al. 2010). Cross-linkers such as $\alpha$-actinin (Laporte et al. 2012) and plastin (Ding et al. 2017) determine the material properties of actomyosin networks. Therefore, the activity, turnover rate and specificity of cross-linkers must be under precise regulation (Levayer and Lecuit 2012). Actomyosin networks are highly dynamic. F-actin severing and depolymerization are essential for the maintenance of actomyosin networks (Chugh and Paluch 2018). The contractility of actomyosin networks is achieved mainly by myosin motor activity, which is under the control of the Rho GTPase signaling pathway, including Rho, Rac and Cdc42 (Guilluy et al. 2011). In addition, F-actin turnover has been suggested to contract actomyosin networks in a myosin-independent manner. For example, in starfish oocytes the chromosomes are transported to the cortex of the egg by actomyosin networks, in which F-actin depolymerization and turnover are indispensable (Bun et al. 2018).

The forces generated by actomyosin networks cannot work alone. To change cell shape and drive cell motion, the actomyosin networks have to anchor to the plasma membrane via adapter molecules (Fig. 2c). For example, during cytokinesis, the actomyosin network is coupled to plasma membrane by ERM proteins and anillin, which is essential for cleavage furrow ingression (Hiruma et al. 2017). In the epithelium, cells are connected by cell-cell junctions, including adherens junctions (AJs). AJs are composed of the transmembrane protein cadherin and the cytosolic protein catenin. Cell-cell adhesion is mediated by $\mathrm{Ca}^{2+}$-dependent homophilic interactions of extracellular cadherin domains. The intracellular domain of cadherin binds to the catenin complex, thus linking to actin filaments. Vinculin binding to $\alpha$-catenin proteins enhances the connection of the actin cytoskeleton to AJs. In this way, AJs transmit forces generated by actomyosin from one cell to another. AJs are not only passive force adopters, but also involved in coordinating cells actively (Rübsam et al. 2018). The actomyosin networks attach to integrins, and therefore the forces generated by actomyosin are exerted against the extracellular matrix (ECM), resulting in cell migration and cell shape change (Schwartz 2010). In some cases, the ECM undergoes remodeling owing to the forces being exerted on them (Haigo and Bilder 2011).

This raises two important questions: How are the AJs and cellular polarity established? How do actomyosin networks drive tissue morphogenesis?

\section{Polarity establishment}

Cells in developing blastula develop distinctly in the apical and the basolateral domains. In parallel, cellular junction complexes are assembled. Generally, the cell-cell contact region is in the basolateral domain, whereas cell contact-free region is in the apical domain (Fig. 3a). Extensive research on Drosophila, C. elegans and mammalian cultured cells has provided us a picture of the molecular regulation of polarity formation and maintenance. Three groups of molecules are required in polarity formation. (1) Par-3/Par-6/aPKC and (2) Crumbs complex, which localize at the apical domain, whereas (3) Scribble complex and Par-1 localize at the basolateral domain. The Scribble complex and Par-1 inhibit the apical proteins from localizing at the basolateral domain. Likewise, Par-3/Par-6/aPKC and Crumbs complex restrict the basolateral localization of Scribble complex and Par1. Such mutually antagonistic interactions between apical and basolateral proteins lead to the formation of polarity (Fig. 3b). The emergence of cell polarity during early embryogenesis in several species has been documented 
A

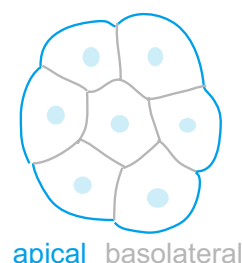

C
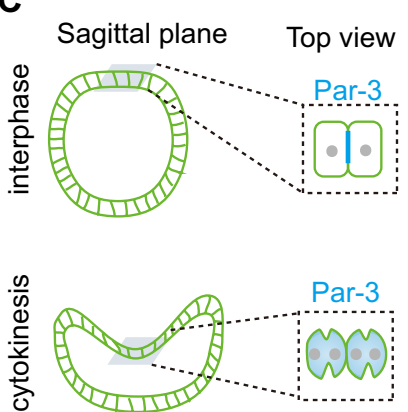

B

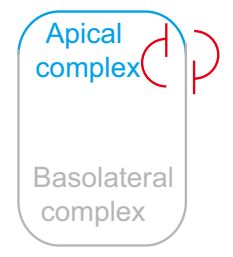

D

before lumen formation

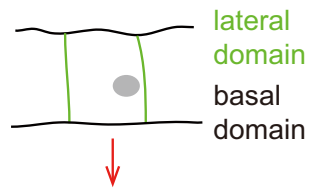

onset of lumen formation

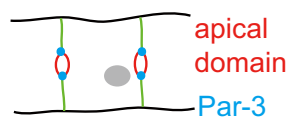

Fig. 3 Polarity establishment. a The apical and basolateral domain of cell in blastula. b Mutually antagonistic interactions between apical and basolateral protein complex leads to the polarity formation and maintenance. c The polarity undergoes "re-establishment"-“de-establishment" cycle along with the cell cleavage cycle in Nematostella. d The polarity emerges during lumen formation in ascidian notochord cells

(Nance 2014). The molecular mechanisms are conserved. However, the regulatory details may differ among species.

The sea anemone Nematostella vectensis displays a chaotic cleavage without a pattern (Fritzenwanker et al. 2007), implying that cell polarity is not yet established at this developmental stage (Salinas-Saavedra et al. 2015). Antibody immunostaining and mRNA injections indicate that different Par proteins show distinct localizations in the gastrula, but not in the cleavage stage (Salinas-Saavedra et al. 2015). Ragkousi et al. (2017) proposed that cell polarity was obtained by the four-cell stage and a primary epithelium was formed by the 16-cell stage based on the localization of Par proteins at the cell-cell contacts.

How is cellular polarity establishment embedded in the fast cleavage cycle in early embryogenesis? A recent study has shown that cell polarity undergoes a re-establishment-de-establishment cycle along with the cell cleavage cycle in Nematostella (Ragkousi et al. 2017). Despite the high variability of cleavage patterns, a coeloblastula with a single layer of epithelial cells is formed after 11 rounds of cleavage. The prominent feature during Nematostella cleavage cycles is that the whole embryo shows interphasic compaction and mitotic decompaction cycles, and such cyclic behavior parallels the re-establishment and de-establishment of apical polarity marked by Par-3 and Par-6 (Ragkousi et al. 2017) (Fig. 3c). These compaction-decompaction cycles are more pronounced later in the blastula, showing a pulsating behavior which occurs between the 128-cell stage and gastrulation. Blastoderms display a spherical shape when the cells are in interphase, whereas they become flattened when the cells are in mitosis. The invagination from a single pole leads to the blastula flattening. Upon the completion of cell cytokinesis, the spherical shape of blastoderms reforms. After several rounds of the spherical-flattened reshaping cycles, the blastula stops pulsating and remains as a spherical shape until the beginning of gastrulation (Fritzenwanker et al. 2007). Pharmacological inhibition of the cell cycle and of the cytoskeletal structure leads to loss of the re-establishment-de-establishment cycle of apical polarity (Ragkousi et al. 2017) and stops the pulsating of the blastula in later stages (Fritzenwanker et al. 2007). It is reasonable to consider that the cellular material properties, e.g., stiffness and tension (Kasza et al. 2007) differ between interphase and interphase, which leads to the blastula flattening. Alternatively, cytokinesis releases the tension and thus the blastula flattens, like a balloon leaking air. Nevertheless, the molecular details of how the cell cycle couples to tissue shape change need to be addressed in future. Another remaining question is how the invagination point is determined. The point of the invagination will be the position where gastrulating invagination takes place. This implies the existence of a genetic or physical constraint in this particular region.

The early embryogenesis of ascidians starts with a series of stereotypical cleavages, giving rise to a compact monolayer ball of cells, which is polarized by expression and localization of Par proteins. In later stage, the AJs assemble and a mature epithelium is organized (Patalano et al. 2006). A striking feature of ascidians is the de novo formation of the apical domain during notochord morphogenesis (Fig. 5d) (Dong et al. 2009). When the notochord cells reach a certain volume, the center of contact between neighboring cells differentiates and acquires apical domain properties. This process is typical polarity establishment. Like in Drosophila embryos and cells in a Petri dish, Par-3/Par-6/aPKC components in the ascidian notochord show polarized distribution, and Par-3 is the key molecule controlling the formation of polarity (Denker et al. 2013) (Fig. 3d). However, the upstream signal regulating RAP-3 is unknown.

\section{Tissue invagination: apical constriction and beyond}

The contraction of apical actomyosin networks, attached to AJs, drives the shrinking of the apical area of the cell, resulting in a cell shape change from columnar to wedge or bottle shape. Apical constriction is wildly used in different developmental contexts, especially in the initiation of gastrulation (Fig. 4). Tissue invagination mediated by apical constriction is a key step of transition from a flat 2-D cell sheet to 3-D 

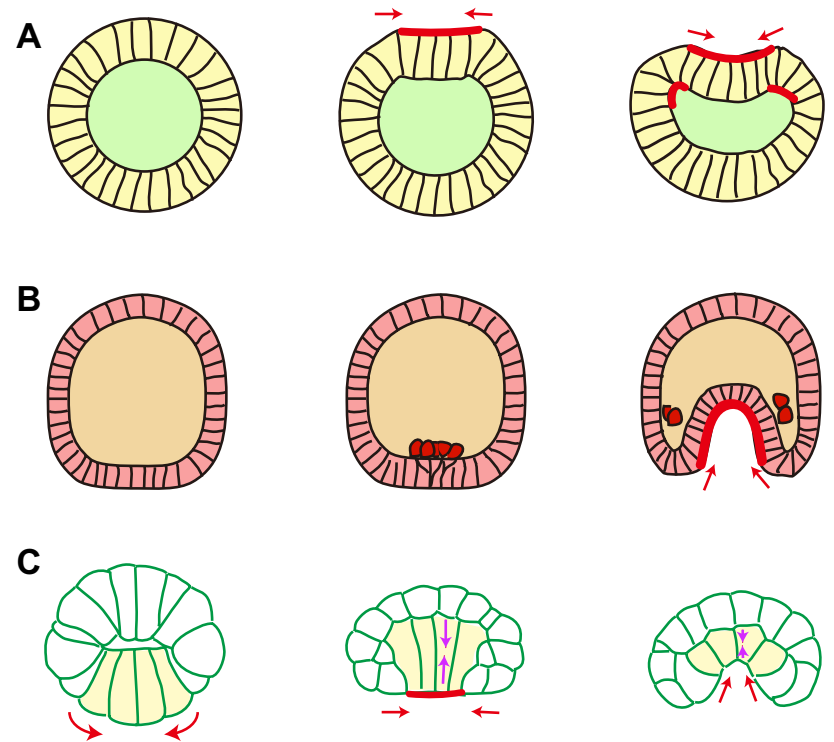

Fig. 4 The apical constriction initiates the gastrulation. a Sea anemone. b Sea urchin. c Ascidian

structure, such as pits and tubes. Being a very widespread process, tissue invagination takes place in all developmental processes, from gastrulation, neurulation to eye cups and renal tubule formation (Martin and Goldstein 2014).

The molecular regulation of apical constriction has been extensively studied in ventral furrow formation during Drosophila melanogaster gastrulation (Coravos and Martin 2016; Martin et al. 2009, 2010; Mason et al. 2013; Vasquez et al. 2014; Xie and Martin 2015). These studies provide a detailed understanding of how apical constriction is achieved and regulated spatially and temporally, and how apical constriction drives tissue to reshape on a multiple cellular level.

At the onset of gastrulation in the sea anemone Nematostella, the animal pole of cells forms a pre-endodermal plate, which then invaginates into the blastocoel via typical apical constriction with the strong accumulation of F-actin at the apical regions of constricting cells (Magie et al. 2007; Tamulonis et al. 2011). Surprisingly, inhibition of myosin activity cannot completely eliminate apical constriction, which suggests the existence of additional mechanisms for this constriction (Pukhlyakova et al. 2018). Shortly after, the blastoporal cells, which surround the pre-endodermal plate, constrict on the basal side and promote further invagination of the pre-endodermal plate. The constriction from the blastoporal cells relies on myosin activity (Pukhlyakova et al. 2018); however, the molecular pathway of this process remains unclear.

During gastrulation of sea urchins, the skeletogenic mesenchyme cells, also called primary mesenchyme cells (PMCs), leave the vegetal region of the spherical blastoderm and ingress into the blastocoel under the control of transcriptional factor snail (Wu and McClay 2007). During the detachment, PMCs undergo apical constriction (Sepúlveda-Ramírez et al. 2018) and membrane remodeling via endocytosis (Wu and McClay 2007). The cells in the vegetal region thicken and flatten, resulting in the formation of the vegetal plate and the shape change of the blastoderm. The vegetal plate invaginates about $1 / 4$ to $1 / 2$ the way into the blastocoel via apical constriction, forming the archenteron (Kimberly and Hardin 1998). After a short pause, the archenteron starts elongating, a process that involves different cellular mechanisms (see below).

Apical constriction is crucial for tissue bending. In addition, there are other mechanisms that contribute to this process. For example, cell mitotic rounding (Kondo and Hayashi 2013) and apoptosis (Monier et al. 2015) are actively involved in tissue invagination in certain developmental contexts. Endoderm invagination in ascidians utilizes apical constriction as the first step. Interestingly, in the next step the apical-basal shortening mechanism drives the bona fide invagination process, as demonstrated both experimentally and by numerical simulations (Sherrard et al. 2010). Surprisingly, the apical-basal shortening relies on Rhoindependent myosin contractility, although the molecular pathway is unknown.

\section{Tissue elongation}

Tissue elongation is another important event during embryogenesis and organogenesis. Tissue elongation can be achieved in different ways such as stretching by external forces, cell intercalation and oriented cell division (Fig. 5a). The protrusive lamellipodia and filopodia in the leading cells (Malinda et al. 1995; Miller et al. 1995) and the migration of neighboring cells (Lye et al. 2015) are the commonly used external forces. Normally, tissue elongation by stretching as a result of external forces is transient (Kong et al. 2017). Subsequent cell rearrangement or cell-oriented division compensates for the tissue strain from the external forces (LeGoff and Lecuit 2015).

In the gastrulation stage of sea urchins, following archenteron invagination, there is a pause prior to archenteron elongation. The dominant driving force of archenteron elongation is cell intercalation, as the number of cells around the circumference of the archenteron decreases (Hardin 1989, 1990) (Fig. 5b).The second contributing factor to archenteron elongation is cell shape change with the thickened cells of the vegetal plate become thinner. However, the molecular regulation of this is not clear yet. The third contributing factor is the filopodia from secondary mesenchyme cells (SMCs) located at the tip of the archenteron. These filopodia extend through the blastocoel, searching the proper positions on the wall of the blastocoel by continually 
Fig. 5 Tissue elongation. a

Three mechanisms used in tissue elongation. b Sea urchin archenteron elongation involves cell intercalation, as shown in color-coded cells, as well as stretching from SMCs protrusion and oriented cell division. c The three phases of ascidian notochord elongation. Cell intercalation is involved in the first phase. In the second phase, the contractile actomyosin ring drives the notochord cell elongating along the A-P axis. In the third phase, luminal pockets enlarge and the notochord cells migrate bi-directionally, leading to the fusion of the luminal pockets, resulting in a long, continuous, single lumen and an elongated notochord
A

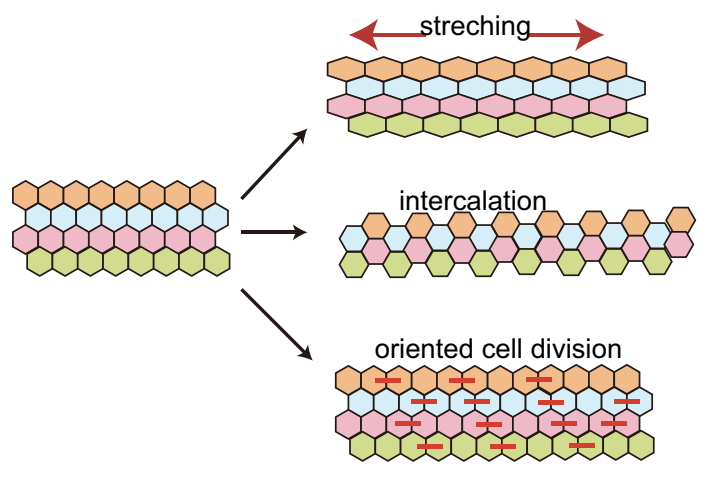

B

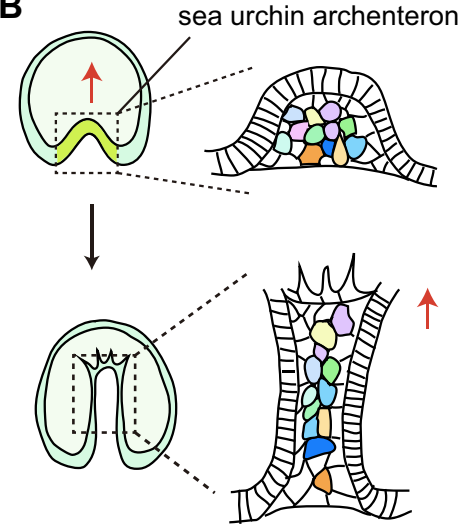

C

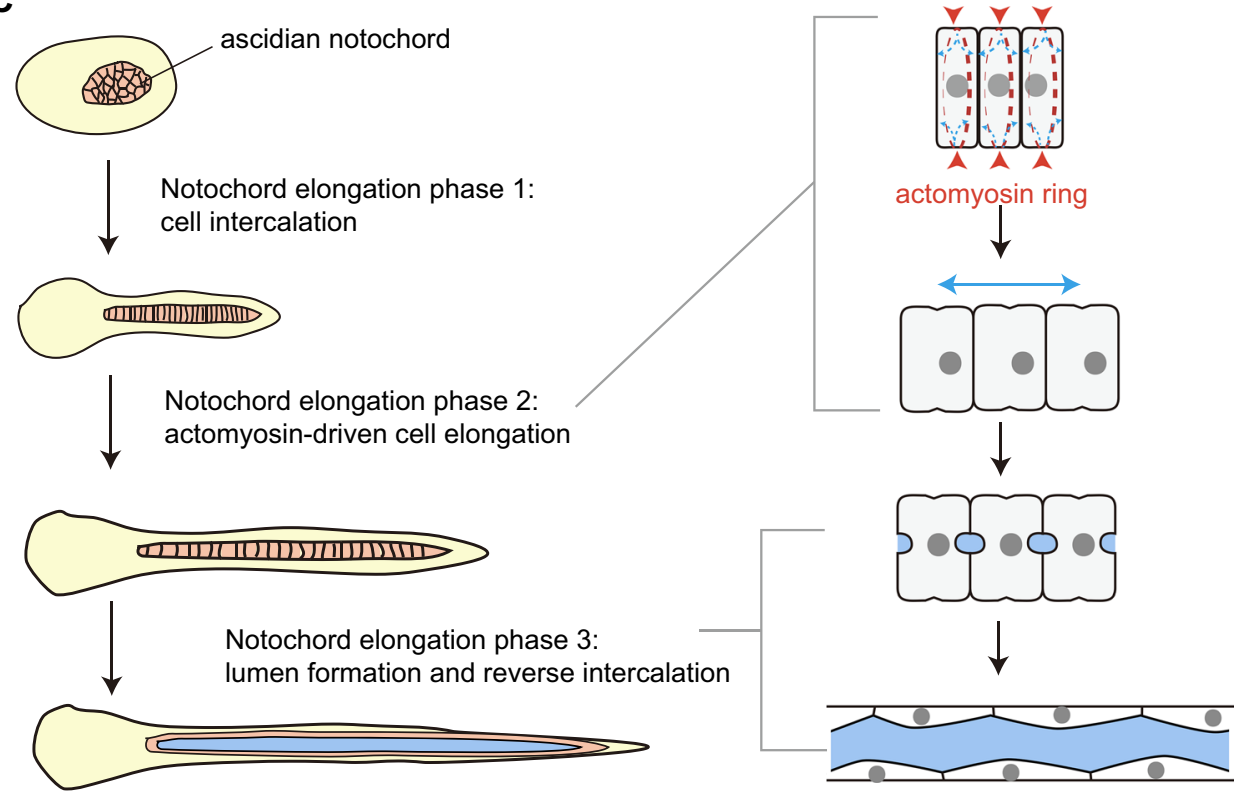

extending and retracting. As soon as the filopodia attach to the right region of the wall, they pull the archenteron inward (Malinda et al. 1995; Miller et al. 1995). A recent study suggested that oriented cell division was also involved in this process (Martik and McClay 2017). These mechanisms work in coordination, driving the archenteron elongation.

The ascidian notochord is an ideal model to study the mechanisms of tissue elongation. Its morphogenesis is well documented (Jiang and Smith 2007; Lu et al. 2019; Smith 2018). The notochord arises from two lineages, the anterior 32 notochord cells derive from blastomeres A7.3, A7.7 and their bilateral partners, and the posterior eight cells derive from blastomere B8.6 and its bilateral partner. After two rounds of mitosis of the blastomeres, the notochord precursor forms a monolayer of 40 cells which will undergo a series of cell shape changes and rearrangements, giving rise to a swimming larva with an elongated tail (Smith 2018). The ascidian notochord elongates in three phases ( $\mathrm{Lu}$ et al. 2019): (1) intercalation of cells drives the notochord to form a monolayer structure with only one row of cells; (2) the cell shapes change leading to the elongation of notochord along the A-P axis; (3) tubulogenesis further lengthens the notochord, resulting in a straight tube that is closed at both ends (Fig. 5c-f).

In the first phase, the notochord precursor cells invaginate into the midline of the embryo via an apical constriction (Munro and Odell 2002). The molecular dynamics in this process is yet to be studied in detail. Subsequently, cells undergo intercalation and deformation in the cylindrical rod, giving rise to an extended notochord along the A-P axis. The 40 cells are disc shaped and aligned end to end with each other giving a "stack of coins" appearance (Fig. 5c). Actinbased protrusions were observed during this process (Munro and Odell 2002). It was subsequently demonstrated that the protrusive activity relies on FGF3 signaling (Shi et al. 2009) and that the orientation of the protrusions depends on PCP signaling (Jiang et al. 2005; Keys et al. 2002). 
In the second phase, the coin-shaped cells increase in length along the A-P axis forming a tambour-shaped structure (Fig. 5c). Experimental studies and physical models indicate that the process is driven by the actomyosin contractile ring (Dong et al. 2011; Sehring et al. 2014). The actomyosin contractile ring is reminiscent of the cytokinetic ring in terms of its compositions and architecture. Nevertheless, the consequences of these two similar ring structures are completely different, with one driving cell elongation and the other separating the parent cell into two daughter cells. Nevertheless, several questions remain to be answered, for example: How does functional differentiation occur in different cellular contexts? Is Rho signaling regulated differently in these two scenarios? Does the material properties of cell cortex matter?

Tubulogenesis takes place during the third phase. After the cells form tambour shape, a novel luminal domain (apical domain) emerges at the center of contact between two adjacent notochord cells, which requires the Par-3/Par-6/ aPKC system (Denker et al. 2013). This process resembles lumen formation in hepatocytes and MDCK cells. The emergence of the new apical domain requires interplay between RhoA and Rac1 activities in MDCK cells (Overeem et al. 2015). Whether similar mechanisms are used in the ascidian notochord needs to be determined by further studies. The apical domain expands with the increase of the extracellular lumen volume, leading to bi-concave notochord cells (Fig. 5e). After lumen growth, the cell surface increases 1.5-fold (Dong et al. 2009). The molecular nature of how to fuel cell surface growth is unclear. Vesicle trafficking is one potential mechanism. Lumen inflation requires transmembrane transport of fluid into the lumen and the power for such transport is an osmotic gradient that originates from the high amount of glycosaminoglycans and ions in the lumen (Denker and Jiang 2012). Anion transporter Ci-Slc26a $\alpha$ is indispensable for lumen expansion, but is not involved in apical/luminal domain specification and biogenesis (Deng et al. 2013). At the onset of lumen pocket formation, cytoplasmic flow in the notochord cells is observed (Mizotani et al. 2018), which might be interpreted as transportation of material for apical membrane growth and lumen inflation. Such cytoplasmic flow requires the interaction of the scaffold protein 14-3-3ea with ERM (ezrin/radixin/moesin) at the basal cortex of the cell. 14-3-3ea and ERM display pulsatile accumulation at the basal cortex, in parallel with the translocation of these two proteins to the luminal domain. Chemical inhibition of 14-3-3ea leads to the disappearance of cytoplasmic flow, thereby hampering lumen formation. This phenotype can be copied by morpholino knock-down of ERM (Mizotani et al. 2018). However, the physical nature underlying the cytoplasmic flow is unknown.

When the lumen pockets reach a certain volume, a highly coordinated cellular rearrangement is triggered via an unknown mechanism, leading to the fusion of separate lumens. At the completion of apical domain expansion, the volume of the extracellular lumen increases resulting in biconcave notochord cells with a deep curvature. At this stage, notochord cells start changing shape dramatically. On one side the anterior and posterior edges retract and move close to each other, whereas on the opposite side the cell extends anteriorly and posteriorly. Neighboring cells undergo the opposite movement. As a result of this collective movement, the anterior and posterior edges meet and adjacent lumens coalesce. New cell-cell contacts are established during this process such that any given notochord cell has six cell-cell contacts instead of the initial two contacts. As a consequence, the number of neighboring cells of a given notochord cell increases from two to four. Due to the similarity with the conventional cell intercalation, this process is called reverse intercalation (Dong et al. 2009). Such coordinated and self-organized cellular behavior prompts the following questions: How do the cells coordinate with each other? Is mechano-transduction process involved? How is the reverse intercalation initiated? Does the reverse intercalation rely on the integrity of tissue? Answering those questions will help us to increase our understanding of tissue elongation and development.

\section{Metamorphosis in ascidians}

Morphogenesis can occur in the post-embryonic stage, for example during metamorphosis in insects, amphibians and fish. Metamorphosis, a process that transforms the larva into an adult, is widespread in the animal kingdom ranging from Porifera to Chordata (Werner 1988). This transformation involves complicated morphogenetic movements and physiological changes, which depend on the degree of difference between the two forms. For example, salamanders undergo only minor changes in body plan, whereas insects and tunicates experience more radical change. An intriguing question is how these cellular events, mechanical forces and regulatory gene networks are coordinated in space and time as the "old" organs are destroyed and "new" organs are established. Numerous studies based on several animal phyla have demonstrated that metamorphosis is reliant on conserved endocrine hormone signaling (Laudet 2011), such as ecdysones in insects and thyroid hormones (THs) in cephalochordates (Paris et al. 2008), urochordates (Patricolo et al. 2001), teleost fishes (Schreiber and Specker 1998; Yamano and Miwa 1998) and amphibians (Yaoita 2019). Among these, the tail resorption of Xenopus tadpoles is the most spectacular and best-known example of a THs-dependent chordate metamorphic event. Localized morphogenetic and physiological changes in tadpole tissues, such as muscle cell 
death and the growth of hindlimbs, are strongly related to their differential sensitivity to THs level.

Compared with terrestrial animals, less is known about metamorphosis in marine organisms. Many marine invertebrates that undergo metamorphosis, including the corals (Grasso et al. 2011), sea urchins (Cameron and Hinegardner 1978; Rahman et al. 2012) and sea squirts (Theriot and Mitchison 1993), possess a biphasic life history with a pelagic larval and benthic adult phase. Although metamorphosis in most marine invertebrates remains to be explored, tunicates have provided an excellent model. One of the bestknown metamorphotic events in this group is tail regression during the transition from larva to juvenile in ascadians (Borisy and Svitkina 2000). Tail regression of certain ascidian larvae can be completed within 15-20 min after the initiation of metamorphosis (Rottner and Schaks 2019). The morphogenetic mechanism underlying the remarkable speed of this process has fascinated biologists for years. For example, although the cellular events during the tail regression have been well described (Kamran et al. 2017), the mechanical force that drives the withdrawal of the tail into the trunk remains ambiguous. It has been proposed that the mechanical forces are provided by the contractile notochord, epidermis or muscle tissue (Kominami and Takata 2004). Programmed cell death is also involved in ascidian tail regression and contributes to tail shortening in some species (Munro and Odell 2002). To date, however, there is no direct evidence to show that such a mechanism is sufficient to drive tail regression at the speed observed. By live imaging, genetic perturbations and biophysical modeling, it is becoming possible to elucidate the underlying cellular processes and mechanical basis. Ascidian tail regression is emerging as a new model to study the mechanism of morphogenesis, which might provide new insights into how an organism couples mechano-chemical signals to achieve tissue transformation.

\section{Conclusion and perspectives}

A handful of cellular mechanisms and biophysical constraints give rise to a broad developmental phenomenon, embedded in different developmental contexts. For example, cell apical constriction mediated by actomyosin contractility is involved in most tissue-bending processes, and cell intercalation occurs in most tissue elongation processes. This prompts an interesting question: How do these animals use similar toolboxes to generate such different forms of life? To answer this question, we need data not only on well-established model organisms such as Drosophila and C. elegans, but also other, especially marine, animals which show a striking diversity of developmental morphologies. Significant progress on marine animal developmental research in recent years has expanded our understanding. For example, a recent study found that the Hox gene is involved in axial identities in the developing embryo of sea anemones and proposed that the axial Hox code may have controlled body patterning before the evolution of the bilaterian A-P axis (He et al. 2018). The second example is the discovery of reverse intercalation in ascidian notochord morphogenesis as a novel strategy to form a tubular organ (Dong et al. 2009). Subsequently, this type of tubulogenesis is also observed in zebrafish vascular anastomosis (Herwig et al. 2011) and gut formation (Alvers et al. 2014), suggesting that this novel mechanism of morphogenesis found in marine animals is highly conserved. Many questions remain unanswered, for example, the mechanisms of cell coordination in ascidian notochord morphogenesis, and the molecular pathway to link tissue invagination and cell cycle in the sea anemone blastula. Knowledge and tools developed in Drosophila and other model organisms, together with the striking features of marine animals, will contribute much to our understanding of basic biological processes.

Acknowledgements The research work in BD's laboratory was supported by the Marine S\&T Fund of Shandong Province for Pilot National Laboratory for Marine Science and Technology (Qingdao) (Nos. 2018SDKJ0302-1, 2018SDKJ0406-5), the Fundamental Research Funds for the Central Universities under Grant (201822016), and the Taishan Scholar Program of Shandong Province, China (201502035). ZL was supported by Deutsche Forschungsgemeinschaft (DFG, German Research Foundation, SFB-937/A10).

Author contributions $\mathrm{ZL}$ and BD conceptualized the framework of this review. QL wrote "Metamorphosis in ascidian". ZL wrote the main body. BD and ZL reviewed and edited the article.

\section{Compliance with ethical standards}

Conflict of interest All the authors declare that they have no conflict of interest.

Animal and human rights statement This article does not contain any studies with human participants or animals performed by any of the authors.

Open Access This article is distributed under the terms of the Creative Commons Attribution 4.0 International License (http://creativeco mmons.org/licenses/by/4.0/), which permits unrestricted use, distribution, and reproduction in any medium, provided you give appropriate credit to the original author(s) and the source, provide a link to the Creative Commons license, and indicate if changes were made.

\section{References}

Abitua PB, Gainous TB, Kaczmarczyk AN, Winchell CJ, Hudson C, Kamata K, Nakagawa M, Tsuda M, Kusakabe TG, Levine M (2015) The pre-vertebrate origins of neurogenic placodes. Nature 542:462-465 
Abreu-Blanco MT, Verboon JM, Liu R, Watts JJ, Parkhurst SM (2012) Drosophila embryos close epithelial wounds using a combination of cellular protrusions and an actomyosin purse string. J Cell Sci 125:5984-5997

Alvers AL, Ryan S, Scherz PJ, Huisken J, Bagnat M (2014) Single continuous lumen formation in the zebrafish gut is mediated by smoothened-dependent tissue remodeling. Development 141:1110-1119

Averbukh I, Ben-Zvi D, Mishra S, Barkai N (2014) Scaling morphogen gradients during tissue growth by a cell division rule. Development 141:2150-2156

Bardet P-L, Guirao B, Paoletti C, Serman F, Léopold V, Bosveld F, Goya Y, Mirouse V, Graner F, Bellaïche Y (2013) PTEN controls junction lengthening and stability during cell rearrangement in epithelial tissue. Dev Cell 25:534-546

Borisy GG, Svitkina TM (2000) Actin machinery: pushing the envelope. Curr Opin Cell Biol 12:104-112

Brubacher JL, Huebner E (2009) Development of polarized female germline cysts in the polychaete, Ophryotrocha labronica. J Morphol 270:413-429

Bun P, Dmitrieff S, Belmonte JM, Nédélec FJ, Lénárt P (2018) A disassembly-driven mechanism explains F-actin-mediated chromosome transport in starfish oocytes. eLife 7:470

Cameron RA, Hinegardner RT (1978) Early events in sea urchin metamorphosis, description and analysis. J Morphol 157:21-31

Chugh P, Paluch EK (2018) The actin cortex at a glance. J Cell Sci $131: j \operatorname{cs} 186254$

Coravos JS, Martin AC (2016) Apical sarcomere-like actomyosin contracts nonmuscle Drosophila epithelial cells. Dev Cell 39:346-358

Costello MJ, Chaudhary C (2017) Marine biodiversity, biogeography, deep-sea gradients, and conservation. Curr Biol 27:R511-R527

Dehal P, Satou Y, Campbell RK, Chapman J, Degnan B, De Tomaso A, Davidson B, Di Gregorio A, Gelpke M, Goodstein DM, Harafuji N, Hastings KEM, Ho I, Hotta K, Huang W, Kawashima T, Lemaire P, Martinez D, Meinertzhagen IA, Necula S et al (2002) The draft genome of Ciona intestinalis: insights into chordate and vertebrate origins. Science 298:2157-2167

Deng W, Nies F, Feuer A, Bočina I, Oliver D, Jiang D (2013) Anion translocation through an Slc26 transporter mediates lumen expansion during tubulogenesis. Proc Natl Acad Sci USA 110:14972-14977

Denker E, Jiang D (2012) Ciona intestinalis notochord as a new model to investigate the cellular and molecular mechanisms of tubulogenesis. Semin Cell Dev Biol 23:308-319

Denker E, Bočina I, Jiang D (2013) Tubulogenesis in a simple cell cord requires the formation of bi-apical cells through two discrete Par domains. Development 140:2985-2996

Ding WY, Ong HT, Hara Y, Wongsantichon J, Toyama Y, Robinson RC, Nédélec F, Zaidel-Bar R (2017) Plastin increases cortical connectivity to facilitate robust polarization and timely cytokinesis. J Cell Biol 216:1371-1386

Dong B, Horie T, Denker E, Kusakabe T, Tsuda M, Smith WC, Jiang D (2009) Tube formation by complex cellular processes in Ciona intestinalis notochord. Dev Biol 330:237-249

Dong B, Deng W, Jiang D (2011) Distinct cytoskeleton populations and extensive crosstalk control Ciona notochord tubulogenesis. Development 138:1631-1641

Fehon RG, McClatchey AI, Bretscher A (2010) Organizing the cell cortex: the role of ERM proteins. Nat Rev Mol Cell Biol 11:276-287

Fritzenwanker JH, Genikhovich G, Kraus Y, Technau U (2007) Early development and axis specification in the sea anemone Nematostella vectensis. Dev Biol 310:264-279

Gilmour D, Rembold M, Leptin M (2017) From morphogen to morphogenesis and back. Nature 541:311-320
Grasso LC, Negri AP, Foret S, Saint R, Hayward DC, Miller DJ, Ball EE (2011) The biology of coral metamorphosis: molecular responses of larvae to inducers of settlement and metamorphosis. Dev Biol 353:411-419

Guilluy C, Garcia-Mata R, Burridge K (2011) Rho protein crosstalk: another social network? Trends Cell Biol 21:718-726

Gustafson T, Wolpert L (1961) Studies on the cellular basis of morphogenesis in the sea urchin embryo: gastrulation in vegetalized larvae. Exp Cell Res 22:437-449

Haigo SL, Bilder D (2011) Global tissue revolutions in a morphogenetic movement controlling elongation. Science 331:1071-1074

Hardin J (1989) Local shifts in position and polarized motility drive cell rearrangement during sea urchin gastrulation. Dev Biol 136:430-445

Hardin J (1990) Context-sensitive cell behaviors during gastrulation. Sem Dev Biol 1:335-345

He S, del Viso F, Chen C-Y, Ikmi A, Kroesen AE, Gibson MC (2018) An axial Hox code controls tissue segmentation and body patterning in Nematostella vectensis. Science 361:1377-1380

Heisenberg C-P, Bellaïche Y (2013) Forces in tissue morphogenesis and patterning. Cell 153:948-962

Herwig L, Blum Y, Krudewig A, Ellertsdottir E, Lenard A, Belting H-G, Affolter M (2011) Distinct cellular mechanisms of blood vessel fusion in the zebrafish embryo. Curr Biol 21:1942-1948

Hiruma S, Kamasaki T, Otomo K, Nemoto T, Uehara R (2017) Dynamics and function of ERM proteins during cytokinesis in human cells. FEBS Lett 591:3296-3309

Izzard CS (1974) Contractile filopodia and in vivo cell movement in the tunic of the ascidian, Botryllus schlosseri. J Cell Sci 15:513-535

Jiang D, Smith WC (2007) Ascidian notochord morphogenesis. Dev Dyn 236:1748-1757

Jiang D, Munro EM, Smith WC (2005) Ascidian prickle regulates both mediolateral and anterior-posterior cell polarity of notochord cells. Curr Biol 15:79-85

Johnson AB, Fogel NS, Lambert JD (2019) Growth and morphogenesis of the gastropod shell. Proc Natl Acad Sci USA 116:6878-6883

Kamran Z, Zellner K, Kyriazes H, Kraus CM, Reynier J-B, Malamy JE (2017) In vivo imaging of epithelial wound healing in the cnidarian Clytia hemisphaerica demonstrates early evolution of purse string and cell crawling closure mechanisms. BMC Dev Biol 17:17

Kasza KE, Rowat AC, Liu J, Angelini TE, Brangwynne CP, Koenderink GH, Weitz DA (2007) The cell as a material. Curr Opin Cell Biol 19:101-107

Keys DN, Levine M, Harland RM, Wallingford JB (2002) Control of intercalation is cell-autonomous in the notochord of Ciona intestinalis. Dev Biol 246:329-340

Kimberly EL, Hardin J (1998) Bottle cells are required for the initiation of primary invagination in the sea urchin embryo. Dev Biol 204:235-250

Kölsch V, Seher T, Fernandez-Ballester GJ, Serrano L, Leptin M (2007) Control of Drosophila gastrulation by apical localization of adherens junctions and RhoGEF2. Science 315:384-386

Kominami T, Takata H (2004) Gastrulation in the sea urchin embryo: a model system for analyzing the morphogenesis of a monolayered epithelium. Dev Growth Differ 46:309-326

Kondo T, Hayashi S (2013) Mitotic cell rounding accelerates epithelial invagination. Nature 494:125-129

Kong D, Wolf F, Grosshans J (2017) Forces directing germ-band extension in Drosophila embryos. Mech Dev 144:11-22

Kornberg TB, Roy S (2014) Cytonemes as specialized signaling filopodia. Development 141:729-736

Kraus Y, Technau U (2006) Gastrulation in the sea anemone Nematostella vectensis occurs by invagination and immigration: an ultrastructural study. Dev Genes Evol 216:119-132 
Laporte D, Ojkic N, Vavylonis D, Wu J-Q (2012) $\alpha$-Actinin and fimbrin cooperate with myosin II to organize actomyosin bundles during contractile-ring assembly. Mol Biol Cell 23:3094-3110

Laudet V (2011) The origins and evolution of vertebrate metamorphosis. Cur Biol 21:R726-R737

Lawrence PA (2001) Morphogens: how big is the big picture? Nat Cell Biol 3:E151-E154

LeGoff L, Lecuit T (2015) Mechanical forces and growth in animal tissues. Cold Spring Harb Perspect Biol 8:a019232

Lemaire P (2011) Evolutionary crossroads in developmental biology: the tunicates. Development 138:2143-2152

Levayer R, Lecuit T (2012) Biomechanical regulation of contractility: spatial control and dynamics. Trends Cell Biol 22:61-81

Lu Q, Bhattachan P, Dong B (2019) Ascidian notochord elongation. Dev Biol 448:147-153

Lye CM, Blanchard GB, Naylor HW, Muresan L, Huisken J, Adams RJ, Sanson B (2015) Mechanical coupling between endoderm invagination and axis extension in Drosophila. PLoS Biol 13:e1002292

Magie CR, Daly M, Martindale MQ (2007) Gastrulation in the cnidarian Nematostella vectensis occurs via invagination not ingression. Dev Biol 305:483-497

Malinda KM, Fisher GW, Ettensohn CA (1995) Four-dimensional microscopic analysis of the filopodial behavior of primary mesenchyme cells during gastrulation in the sea urchin embryo. Dev Biol 172:552-566

Mammoto T, Ingber DE (2010) Mechanical control of tissue and organ development. Development 137:1407-1420

Martik ML, McClay DR (2017) New insights from a high-resolution look at gastrulation in the sea urchin, Lytechinus variegatus. Mech Dev 148:3-10

Martin AC, Goldstein B (2014) Apical constriction: themes and variations on a cellular mechanism driving morphogenesis. Development 141:1987-1998

Martin AC, Kaschube M, Wieschaus EF (2009) Pulsed contractions of an actin-myosin network drive apical constriction. Nature 457:495-499

Martin AC, Gelbart M, Fernandez-Gonzalez R, Kaschube M, Wieschaus EF (2010) Integration of contractile forces during tissue invagination. J Cell Biol 188:735-749

Mason FM, Tworoger M, Martin AC (2013) Apical domain polarization localizes actin-myosin activity to drive ratchet-like apical constriction. Nat Cell Biol 15:926-936

McClay DR (2011) Evolutionary crossroads in developmental biology: sea urchins. Development 138:2639-2648

McDougall C, Degnan BM (2018) The evolution of mollusc shells. Wiley Interdiscip Rev Dev Biol 7:e313

Miller J, Fraser SE, McClay D (1995) Dynamics of thin filopodia during sea urchin gastrulation. Development 121:2501-2511

Mizotani Y, Suzuki M, Hotta K, Watanabe H, Shiba K, Inaba K, Tashiro E, Oka K, Imoto M (2018) 14-3-3ea directs the pulsatile transport of basal factors toward the apical domain for lumen growth in tubulogenesis. Proc Natl Acad Sci USA 115:E8873-E8881

Monier B, Pélissier-Monier A, Brand AH, Sanson B (2010) An actomyosin-based barrier inhibits cell mixing at compartmental boundaries in Drosophila embryos. Nat Cell Biol 12:60-65

Monier B, Gettings M, Gay G, Mangeat T, Schott S, Guarner A, Suzanne M (2015) Apico-basal forces exerted by apoptotic cells drive epithelium folding. Nature 518:245-248

Munro EM, Odell GM (2002) Polarized basolateral cell motility underlies invagination and convergent extension of the ascidian notochord. Development 129:13-24

Munro E, Nance J, Priess JR (2004) Cortical flows powered by asymmetrical contraction transport PAR proteins to establish and maintain anterior-posterior polarity in the early C. elegans embryo. Dev Cell 7:413-424
Nance J (2014) Getting to know your neighbor: cell polarization in early embryos. J Cell Biol 206:823-832

Nüsslein-Volhard C, Wieschaus E (1980) Mutations affecting segment number and polarity in Drosophila. Nature 287:795-801

Overeem AW, Bryant DM, van IJzendoorn SCD (2015) Mechanisms of apical-basal axis orientation and epithelial lumen positioning. Trends Cell Biol 25:476-485

Paris M, Escriva H, Schubert M, Brunet F, Brtko J, Ciesielski F, Roecklin D, Vivat-Hannah V, Jamin EL, Cravedi J-P, Scanlan TS, Renaud J-P, Holland ND, Laudet V (2008) Amphioxus postembryonic development reveals the homology of chordate metamorphosis. Curr Biol 18:825-830

Patalano S, Prulière G, Prodon F, Paix A, Dru P, Sardet C, Chenevert J (2006) The aPKC-PAR-6-PAR-3 cell polarity complex localizes to the centrosome attracting body, a macroscopic cortical structure responsible for asymmetric divisions in the early ascidian embryo. J Cell Sci 119:1592-1603

Patricolo E, Cammarata M, D'Agati P (2001) Presence of thyroid hormones in ascidian larvae and their involvement in metamorphosis. J Exp Zool 290:426-430

Pollard TD, Cooper JA (2009) Actin, a central player in cell shape and movement. Science 326:1208-1212

Pukhlyakova E, Aman AJ, Elsayad K, Technau U (2018) $\beta$-Catenindependent mechanotransduction dates back to the common ancestor of Cnidaria and Bilateria. Proc Natl Acad Sci USA 115:6231-6236

Ragkousi K, Marr K, McKinney S, Ellington L, Gibson MC (2017) Cell-cycle-coupled oscillations in apical polarity and intercellular contact maintain order in embryonic epithelia. Curr Biol 27:1381-1386

Rahman MA, Yusoff FM, Arshad A, Shamsudin MN, Amin SMN (2012) Embryonic, larval, and early juvenile development of the tropical sea urchin, Salmacis sphaeroides (Echinodermata: Echinoidea). Sci World J 2012:1-9

Rauzi M, Lenne P-F, Lecuit T (2010) Planar polarized actomyosin contractile flows control epithelial junction remodelling. Nature 468:1110-1114

Reeves WM, Wu Y, Harder MJ, Veeman MT (2017) Functional and evolutionary insights from the Ciona notochord transcriptome. Development 144:3375-3387

Rottner K, Schaks M (2019) Assembling actin filaments for protrusion. Curr Opin Cell Biol 56:53-63

Rübsam M, Broussard JA, Wickström SA, Nekrasova O, Green KJ, Niessen CM (2018) Adherens junctions and desmosomes coordinate mechanics and signaling to orchestrate tissue morphogenesis and function: an evolutionary perspective. Cold Spring Harb Perspect Biol 10:a029207

Salinas-Saavedra M, Stephenson TQ, Dunn CW, Martindale MQ (2015) Par system components are asymmetrically localized in ectodermal epithelia, but not during early development in the sea anemone Nematostella vectensis. EvoDevo 6:20

Schreiber AM, Specker JL (1998) Metamorphosis in the summer flounder (Paralichthys dentatus): stage-specific developmental response to altered thyroid status. Gen Comp Endocrinol 111:156-166

Schwartz MA (2010) Integrins and extracellular matrix in mechanotransduction. Cold Spring Harb Perspect Biol 2:a005066

Sea Urchin Genome Sequencing Consortium, Sodergren E, Weinstock GM, Davidson EH, Cameron RA, Gibbs RA, Angerer RC, Angerer LM, Arnone MI, Burgess DR, Burke RD, Coffman JA, Dean M, Elphick MR, Ettensohn CA, Foltz KR, Hamdoun A, Hynes RO, Klein WH, Marzluff W et al (2006) The genome of the sea urchin Strongylocentrotus purpuratus. Science 314:941-952 
Sehring IM, Dong B, Denker E, Bhattachan P, Deng W, Mathiesen BT, Jiang D (2014) An equatorial contractile mechanism drives cell elongation but not cell division. PLoS Biol 12:e1001781

Sepúlveda-Ramírez SP, Toledo-Jacobo L, Henson JH, Shuster CB (2018) Cdc42 controls primary mesenchyme cell morphogenesis in the sea urchin embryo. Dev Biol 437:140-151

Sherrard K, Robin F, Lemaire P, Munro E (2010) Sequential activation of apical and basolateral contractility drives ascidian endoderm invagination. Curr Biol 20:1499-1510

Shi W, Peyrot SM, Munro E, Levine M (2009) FGF3 in the floor plate directs notochord convergent extension in the Ciona tadpole. Development 136:23-28

Shyer AE, Huycke TR, Lee C, Mahadevan L, Tabin CJ (2015) Bending gradients: how the intestinal stem cell gets its home. Cell 161:569-580

Smith WC (2018) Cellular processes of notochord formation. Adv Exp Med Biol 1029:165-177

Steinmetz PRH, Aman A, Kraus JEM, Technau U (2017) Gut-like ectodermal tissue in a sea anemone challenges germ layer homology. Nat Ecol Evol 1:1535-1542

Stolfi A, Ryan K, Meinertzhagen IA, Christiaen L (2015) Migratory neuronal progenitors arise from the neural plate borders in tunicates. Nature 527:371-374

Tamulonis C, Postma M, Marlow HQ, Magie CR, de Jong J, Kaandorp J (2011) A cell-based model of Nematostella vectensis gastrulation including bottle cell formation, invagination and zippering. Dev Biol 351:217-228
Technau U, Steele RE (2011) Evolutionary crossroads in developmental biology: Cnidaria. Development 138:1447-1458

Theriot JA, Mitchison TJ (1993) The three faces of profilin. Cell 75:835-838

Turing AM (1953) The chemical basis of morphogenesis. Philos Trans R Soc Lond B Biol Sci 273:37-72

Vasquez CG, Tworoger M, Martin AC (2014) Dynamic myosin phosphorylation regulates contractile pulses and tissue integrity during epithelial morphogenesis. J Cell Biol 206:435-450

Werner EE (1988) Size, scaling, and the evolution of complex life cycles. In: Ebenman B, Persson L (eds) Size-structured populations. Springer, Berlin, pp 60-81

Wu S-Y, McClay DR (2007) The Snail repressor is required for PMC ingression in the sea urchin embryo. Development 134:1061-1070

Xie S, Martin AC (2015) Intracellular signalling and intercellular coupling coordinate heterogeneous contractile events to facilitate tissue folding. Nat Commun 6:7161

Yamano K, Miwa S (1998) Differential gene expression of thyroid hormone receptor $\alpha$ and $\beta$ in fish development. Gen Comp Endocrinol 109:75-85

Yaoita Y (2019) Tail resorption during metamorphosis in Xenopus tadpoles. Front Endocrinol 10:143 